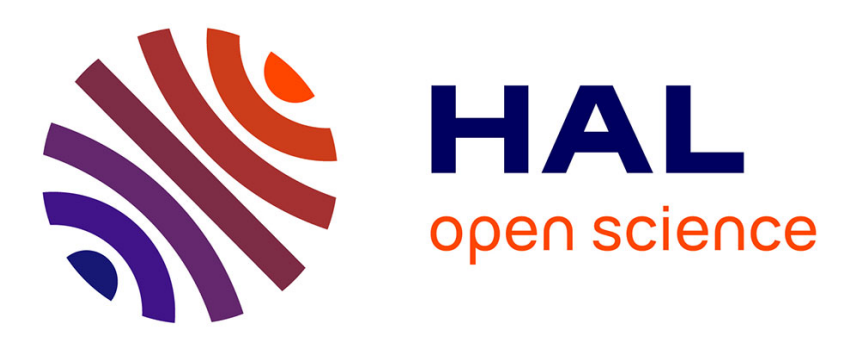

\title{
Systematic Hamiltonian approach to Eden model of cluster growth
}

Y. Shapir, Y.-C. Zhang

\section{To cite this version:}

Y. Shapir, Y.-C. Zhang. Systematic Hamiltonian approach to Eden model of cluster growth. Journal de Physique Lettres, 1985, 46 (12), pp.529-533. 10.1051/jphyslet:019850046012052900 . jpa00232555

\section{HAL Id: jpa-00232555 https://hal.science/jpa-00232555}

Submitted on 1 Jan 1985

HAL is a multi-disciplinary open access archive for the deposit and dissemination of scientific research documents, whether they are published or not. The documents may come from teaching and research institutions in France or abroad, or from public or private research centers.
L'archive ouverte pluridisciplinaire HAL, est destinée au dépôt et à la diffusion de documents scientifiques de niveau recherche, publiés ou non, émanant des établissements d'enseignement et de recherche français ou étrangers, des laboratoires publics ou privés. 


\title{
Classification
}

Physics Abstracts

$02.50-05.70 \mathrm{~L}-68.70$

\section{Systematic Hamiltonian approach to Eden model of cluster growth}

\author{
Y. Shapir and Y.-C. Zhang \\ Physics Department, Brookhaven National Laboratory, Upton, New York 11973, U.S.A.
}

(Reçu le 15 avril 1985, accepté le 26 avril 1985)

\begin{abstract}
Résumé. - Nous présentons une dérivation systématique d'un Hamiltonien pour engendrer toutes les histoires possibles de la croissance d'un amas dans le modèle d'Eden. La généralisation du modèle permettant l'addition de plusieurs nouvelles particules à chaque intervalle de temps est aussi considérée. Les amas d'Eden sont obtenus dans la limite des intervalles infinitésimaux où la densité de nouvelles particules est arbitrairement petite. La théorie du champ, locale dans l'espace mais non Markovienne, est obtenue dans cette limite continue.
\end{abstract}

\begin{abstract}
A systematic Hamiltonian approach to generate all possible histories along which Eden clusters may grow is presented. The generalization of the model which allow the addition of several new particles at each time step is considered. Eden clusters are recovered in the limit of vanishing time intervals where the density of new particles may be made arbitrarily small. The field-theoretic formulation is derived in this continuum limit. It is local in space but not local (non-Markovian) in time.
\end{abstract}

Much interest has been given recently to growth and aggregation processes [1]. This renewed interest was stimulated by the introduction of the Witten-Sander [2] model for diffusion-limited aggregation (DLA). Most of these recent works are numerical simulations of different variants of particles and/or cluster aggregations. Universal features such as the fractal, spectral and spreading dimensionalities are extracted from the aggregates precipitated in these computer experiments. First analytic approaches were based on mean-field [3-5] and Flory type approximations [6].

On a Cayley tree $[7,8]$ the DLA process is equivalent to a simpler model for cluster growth introduced many years ago by Eden [9]. Although this relation holds due to the special topology of the tree, the exact solution of the Eden model [7, 8] on this lattice may provide a starting point to approach both models in lower dimensions as well [7]. Indeed, this unrealistic lattice is equivalent to the limit of infinite dimensionality and has been very useful in the study of many statistical models since it reproduces (and, in some respects, even improves) their mean-field theory. Therefore a field-theory for the Eden model, interesting by itself, may also provide a zerothapproximation to the DLA process. A first step in that direction has been proposed recently by Parisi and Zhang [10]. They suggested the Reggeon field-theory, which describes other Markovian [11] and non-Markovian [12] processes, as a candidate for representing the Eden model as well. They related them through a hierarchy of equations-of-motion which is proposed to describe the Eden mechanism. The resultant Reggeon theory is in the supercritical regime namely deep in the ordered phase. 
In the present communication we derive independently a Hamiltonian operator formalism which systematically generates all Eden clusters (differentiated according to their histories) and from which all averaged properties may be derived. The process is then generalized and described by a local field-theory. This field-theory is explicitly non-Markovian and therefore not trivially susceptible to standard methods of investigation.

To remind the reader, in this process each of the particles on the edge of the cluster may give birth to a new particle on one of its nearest-neighbour unoccupied (perimeter) sites. As defined originally by Eden [9] the related probability is weighted according to the number of particles which may give birth to the new particle (although other definitions exist as well). So the independent random entities are perimeter " dimers " which lie on the links connecting occupied and empty sites. At each time step one of the dimers is chosen randomly and a new particle is added at the empty site of this dimer. In low dimensions $(d=2$ and $d=3)$ the clusters so generated are almost compact with trivial fractal dimension equal to the spatial one (see, e.g., Ref. [13] for a slightly different variant). The situation is less clear in high dimensions [7].

Our approach follows the similar one successfully applied to static dimer models $[11,14,15]$. We literally " construct " the Eden clusters, step by step, with two basic " building block " commuting variables : (i) The " particle " variables : $a_{i}(t)$ which represents a particle deposited on site $i$ at time $t$ (both spatial and temporal coordinates are discretized). They obey $\operatorname{Tr} a_{i}(t) \equiv 1$ and $\operatorname{Tr} a_{i}(t) a_{i}\left(t^{\prime}\right) \equiv 0$ for any $t$ and $t^{\prime}$ (to prevent the occupation of a site by more than one particle). (ii) The " gluon " variables : $P_{i}(t)$ which obey $\operatorname{Tr} P_{i}(t) \equiv 0$ but $\operatorname{Tr} P_{i}(t) a_{i}\left(t^{\prime}\right) \equiv 1$ if $t \geqslant t^{\prime}$ and zero otherwise. A product $\prod_{m} P_{i}\left(t_{m}\right)$ will follow the same rules as applied to the earliest $P_{i}\left(t_{1}\right)$ with $t_{1}=\min \left\{t_{m}\right\}$. This is in order to allow the sticking of more than one nearest-neighbour particle to an already present one.

The evolution operator (later to be related to the Hamiltonian) is :

$$
L(t)=\sum_{\langle i j\rangle}\left\{a_{i}(t) P_{j}(t-1)+a_{j}(t) P_{i}(t-1)\right\}
$$

where the sum run over all nearest-neighbour pairs on any $d$-dimensional lattice.

Let us describe explicitly the first steps in that construction : at time $t=0$ a « seed " particle is present at the origin and denoted by $a_{0}(0)$. At time $t=1$ we apply the evolution operator $L(1)$ and we denote by $W(1)$ all possible wards (products of the variables) so generated :

$$
W(1)=L(1) W(0)
$$

where we have identified $W(0) \equiv a_{0}(0)$. We distinguish among two classes of wards included in $W(1)$ according to their contribution to

$$
S_{2}=\operatorname{Tr} W(1) .
$$

Most of the wards will make vanishing contributions. The only ones whose trace will be unity have the following form :

$$
C(1)=\sum_{\langle j\rangle_{0}} a_{j}(1) P_{0}(0) a_{0}(0)
$$

where $j$ is nearest-neighbour of the origin. Or those are precisely representing all possible Eden clusters with two particles ! We denote $C(1)$ as the nontrivial subset of $W(1)$ of all wards which correspond to Eden clusters.

At the next time step $t=2$ the evolution operator is applied again :

$$
W(2)=L(2) W(1) \text {. }
$$


The subset $C(2) \subset W(2)$ contains all wards with nonvanishing traces which may be constructed from all configurations in $C(1)$. So each cluster of $C(1)$ is an independent source for the elements of $C(2)$ which correspond, one-to-one, to all possible path histories along which a cluster with three particles may be built. The same process is applied iteratively to grow larger and larger clusters. With each different history which leads to a cluster with $n=\tau+1$ particles is associated one element of $C(\tau)$, a subset of :

$$
W(\tau)=\prod_{t=1}^{\tau} L(t) a_{0}(0)
$$

such that :

$$
C(\tau)=\{w(\tau): w(\tau) \in W(\tau), \operatorname{Tr} w(\tau)=1\}
$$

It is straightforward then to extract all possible information on these dynamical clusters. As typical examples we mention :

a) The total number of Eden clusters (distinguished according to their history) with $n=\tau+1$ particles is simply :

$$
S_{n}=\operatorname{Tr} C(\tau)=\operatorname{Tr} W(\tau)
$$

b) The probability for a site $j$ to be occupied at time $t$ is :

$$
\rho(j, t)=\operatorname{Tr} P_{j}(t) W(t) / \operatorname{Tr} W(t) .
$$

c) The joint probability for sites $j_{1}, \ldots, j_{m}$ to be occupied at times $t_{1} \leqslant t_{2} \ldots \leqslant t_{m}$ is :

$$
\rho\left(j_{1}, t_{1} ; \ldots ; j_{m}, t_{m}\right)=\operatorname{Tr} P_{j_{1}}\left(t_{1}\right) \ldots P_{j_{m}}\left(t_{m}\right) W\left(t_{m}\right) / \operatorname{Tr} W\left(t_{m}\right)
$$

and so on.

Given the simplicity of this approach, its generalization to a field-theory is certainly desired. The next required step is to exponentiate the evolution operator in order to find the associated Hamiltonian. Although formally this may be achieved, it requires the introduction of longrange interactions to prevent the addition of more than one particle at a given time step. The Eden process is, however, manifestly local and should be described faithfully by a local theory. We therefore consider the process which allows for a "dilute gas » of new particles the density of which is controlled by a fugacity parameter $z$. In the continuum limit where the number of time steps is taken to infinity, this fugacity will be taken to zero such that a renormalized fugacity will remain finite (but small). In this limit the process is controlled by independent, uncorrelated, events of the addition of one (or not at all) new particle and is therefore equivalent to the original Eden process. This limit certainly alters the simple relation between the time, which becomes a bare unphysical parameter, and the mass of the cluster (which is like the renormalized time) in terms of which all properties are expressed. These renormalizations are beyond the scope of the present work and here we just sketch the main steps leading to the continuum theory.

We first define the new evolution operator as :

$$
U(t)=\exp \{z L(t)\}
$$

Its successive applications from $t=1$ to $t=\tau$ (starting with the seed at the origin) will generate this time a " grandcanonical » ensemble of histories of clusters which have an (almost) arbitrary number of particles $m$ but each weighted by a factor of $z^{m}$. The corresponding generating function is :

$$
G^{\tau}(z)=\left\langle a_{0}(0)\right\rangle_{z}^{\tau}=\operatorname{Tr}\left\{\exp z \sum_{t=1}^{\tau} L(t)\right\} a_{0}(0)
$$


since the " partition function » is trivially given by :

$$
Z^{\tau}(z)=\operatorname{Tr}\left\{\exp z \sum_{t=1}^{\tau} L(t)\right\} \equiv 1,
$$

and accordingly for other averaged correlations.

The corresponding discrete Lagrangian is

$$
\mathcal{L}^{\tau}(z)=z \sum_{t ; t^{\prime}=1}^{\tau} \sum_{(i j)} a_{i}(t) A_{i j}\left(t, t^{\prime}\right) P_{j}\left(t^{\prime}\right)
$$

where $A_{i j}\left(t, t^{\prime}\right)=1$ if $i$ and $j$ are nearest-neighbours and $t-t^{\prime}=1$ but vanish otherwise. This discrete Lagrangian may be Hubbard-Stratonovitch transformed to a field-theory by the introduction of continuous fields $\hat{\phi}_{i}(t)$ and $\phi_{i}(t)$ conjugate to $a_{i}(t)$ and $P_{i}(t)$ respectively. The trace over the latter may be performed. This generates interaction terms between $\hat{\phi}_{i}(t)$ and arbitrary number $\phi_{i}\left(t^{\prime}\right)$ which are local in space but explicitly nonlocal in time. We write down directly the continuum version obtained by the expansion of $A_{i j}^{-1}\left(t, t^{\prime}\right)$ in terms of gradients and replacement of discrete sums by integrals.

The continuous Lagrangian so obtained is :

$$
\begin{aligned}
\mathcal{L}_{z}^{\tau}(\phi, \hat{\phi})= & -\int_{0}^{\tau} \mathrm{d} t \int \mathrm{d}^{d} x\left\{\alpha \hat{\phi}(x, t) \partial_{t} \phi(x, t)-\beta \hat{\phi}(x, t) \nabla^{2} \phi(x, t)\right. \\
& +\gamma \hat{\phi}(x, t) \phi(x, t)-\hat{\phi}(x, t)-\hat{\phi}(x, t) \int_{t}^{\tau} \phi\left(x, t^{\prime}\right) \mathrm{d} t^{\prime} \\
& \left.-\hat{\phi}(x, t) \int_{t}^{\tau} \phi\left(x, t^{\prime}\right) \mathrm{d} t^{\prime} \int_{t^{\prime}}^{\tau} \phi\left(x, t^{\prime \prime}\right) \mathrm{d} t^{\prime \prime}+\cdots\right\}
\end{aligned}
$$

where $\alpha, \beta$ and $\gamma$ are constants which depend on the lattice structure, on $z$, etc.

This is the field-theoretic formulation we have derived for the generalized Eden mechanism. Averages are also expressed as expectation values of local functions of the fields $\hat{\phi}(x, t)$ and $\phi(x, t)$ along with their time integrals.

In forthcoming works it will be shown how this field-theory may be made Markovian (local in time) and generalized to the DLA process by the introduction of more fields.

\section{Acknowledgments.}

We would like to acknowledge discussions with J. L. Cardy, V. Hakim and T. Witten. This work was supported by the Division of Materials Sciences, U.S. Department of Energy under contract DE-AC02-76CH00016.

\section{References}

[1] See, e.g., Kinetics of Aggregation and Gelation, F. Family and D. P. Landau, ed. (North-Holland, Amsterdam) 1984.

[2] Witten Jr., T. A. and Sander, L. M., Phys. Rev. Lett. 47 (1981) 1400.

[3] Nauenberg, M., Phys. Rev. B 28 (1983) 499.

[4] Nauenberg, M., Richter, R. and Sander, L. M., Phys. Rev. B 28 (1983) 1649. 
[5] Ball, R., Nauenberg, M. and Witten Jr., T. A., I.T.P. Report No. 83-37 (unpublished).

[6] Muthukumar, M., Phys. Rev. Lett. 50 (1983) 839.

[7] Parisi, G. and Zhang, Y.-C., Phys. Rev. Lett. 53 (1984) 1791.

[8] Vannimenus, J., Nickel, B. and Hakim, V., Phys. Rev. B 30 (1984) 391.

[9] Eden, M., 4th Berkeley Symp. on Math. Stat. and Probability IV (1961) 233, F. Neyman, ed. (Berkeley : University of California Press).

[10] Parisi, G. and Zhang, Y.-C., BNL preprint.

[11] Cardy, J. L. and Sugar, R. L., J. Phys. A 13 (1980) L243.

[12] Cardy, J. L., J. Phys. A 16 (1983) L709.

[13] Peters, H. P., Stauffer, D., Hölters, H. P. and Loewenich, K., Z. Phys. B 34 (1979) 399.

[14] Shapir, Y., Phys. Rev. A 28 (1983) 1893.

[15] ShapIR, Y., in Proc. of the conference on Supersymmetry in Physics to appear in Physica D (1985). 